\title{
Intra-uterine implant (GyneFix) lost via intestinal route?
}

\author{
Simone Reuter, Dr. med., MFFP, Clinical Medical Officer, Contraception and Sexual Health Service North Derbyshire, \\ Chesterfield, UK. Srinivasan Krishnamurthy, FRCS, FRCOG, Associate Professor, Department of Obstetrics and Gynaecology, \\ Montreal General Hospital, McGill University, Montreal, Quebec, Canada.
}

Correspondence: Dr Simone Reuter, Contraception and Sexual Health Service, Saltergate Health Centre, Chesterfield S40 1SX, $U K$.

(Accepted $12^{\text {th }}$ January 2001)

The Journal of Family Planning and Reproductive Health Care 2001: 27(3): 159-160

\begin{abstract}
Uterine perforation has long been regarded as a complication of the insertion of an intra-uterine contraceptive device (IUD). The development of modern devices with sophisticated insertion systems as well as advanced training requirements seeks to minimise the risk of adverse insertion incidents for women choosing intrauterine contraception. This case report highlights the continuing need for intra- and post-insertion vigilance as even recent advances in IUD technique and technology do not guarantee risk-free insertion.
\end{abstract}

\section{Key message points \\ - Lost threads need to be followed up quickly \\ - Even modern IUDs with sophisticated insertion techniques may lead to perforation.}

\section{Case report}

A 42-year-old parous woman requested an intra-uterine device (IUD) because she felt unhappy about weight gain on Depo Provera. Previously she had used a Gyne T 380 which had been removed due to pain after 6 months. In view of this she was counselled for a copper intra-uterine implant or IUI (GyneFix) and this was inserted at the end of October 1999 by an experienced doctor after negative swab results had been obtained. The fitting was reported as straightforward. The patient attended after 4 weeks for a check as she had suffered from abdominal pain and cramps since the fitting, as well as slight blood stained discharge. There had been no period, which was considered to be an ongoing effect from having used Depo Provera. A random pregnancy test (Clearview) was negative. At examination there was no thread visible nor palpable, and some abdominal tenderness was noted on bimanual examination. Triple swabs were obtained which were shown to be negative. The patient was counselled on the implications of a lost thread and the need to use other contraception while awaiting an ultrasound examination.

Pelvic ultrasound did not identify an implant in utero, pelvic X-ray showed the GyneFix overlying the left sacroiliac joint in mid-December 1999 (Figure 1). The patient was reviewed at clinic with these results and immediately referred to the gynaecologist for laparoscopic removal of the intra-abdominal device. At this stage the patient recommenced use of Depo Provera.

Routine laparoscopy was undertaken by an experienced consultant (SK) in March 2000. Spontaneous perforation of the uterus in a high fundal position while placing the intrauterine manipulator was described in the operation notes. This did not require any intervention as bleeding settled spontaneously. Despite a methodical search, no device was found in the abdomen, good views having been obtained. Intra-operative $\mathrm{X}$-ray screening did not show any device.

The patient made a good post-operative recovery and underwent further abdominal X-ray prior to discharge.
Figure 1 GyneFix overlying left sacroiliac joint (December 1999)

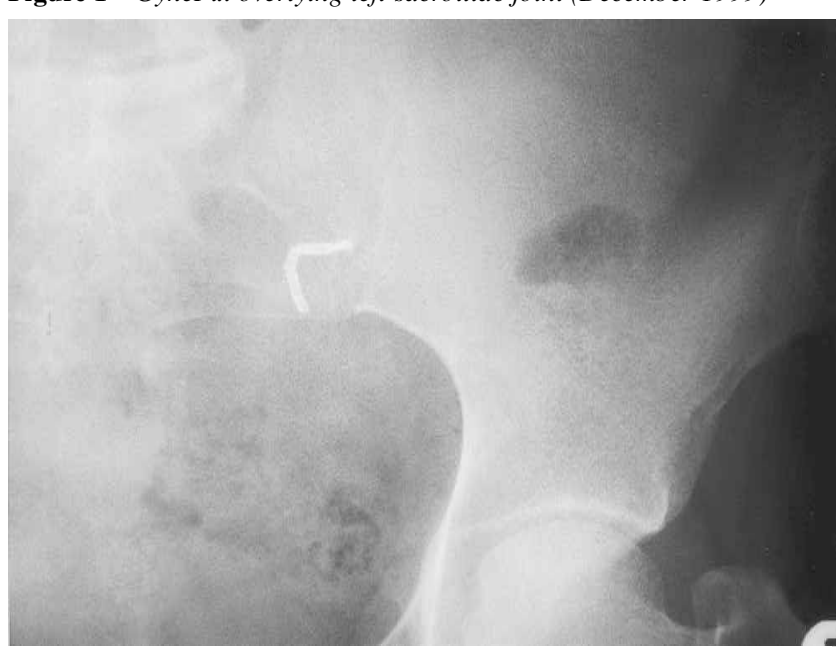

Again no device was seen (Figure 2). The consultant gynaecologist discussed the findings with the patient. She was able to remember a time some weeks prior to admission when she had experienced severe backache and colicky pain. The consultant hypothesised that the device may have eroded into the bowel and been excreted.

Figure 2 GyneFix not present in pelvis (March 2000)

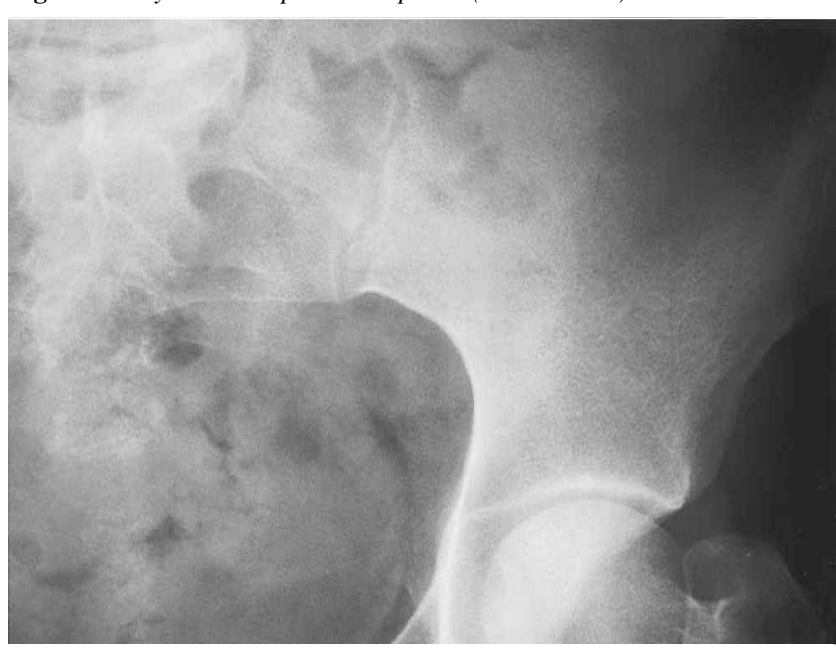

\section{Discussion}

Uterine perforation is a rare but well described hazard of insertion of intra-uterine devices, with a perforation rate of 1.3/1000 insertions reported in large clinical studies. ${ }^{1}$ Perforation is associated with early post partum insertion, ${ }^{2}$ the skill of the inserter, ${ }^{3}$ and potentially with undetected pre-existing uterine pathology. ${ }^{4}$ It has been postulated that in some women myometrial thickness may be decreased predisposing to perforation during insertion of an anchored device. ${ }^{5}$ Use of ultrasound to assess myometrial thickness 
in women prior to GyneFix insertion has been described, ${ }^{4}$ but would make this method unpractical in general practice or family planning clinics. Supervised training in insertion procedures and maintenance of clinical skills are prerequisites for safe practice and the Faculty of Family Planning has mechanisms in place to ensure high standards. ${ }^{6}$

The GyneFix inserter has been designed following studies of myometrial thickness to minimise any risk of primary perforation, even in women with a myometrial thickness at the lower end of the normal range. ${ }^{4}$ Some insertion-related perforations, however, have been reported since its introduction in Europe. In a recent publication, uterine perforation causing acute abdominal pain at insertion followed by ongoing pain was described. The implant was found embedded in the mesorectum and an abscess had formed necessitating laparotomy for removal. ${ }^{7}$ This is in keeping with reports of 'lost' conventional IUDs, which have been commonly found completely or partially embedded in other intra-abdominal structures. ${ }^{8}$ The plastic frames of conventional devices do not lend themselves to complete penetration of other structures and can therefore cause substantial damage and discomfort to women.

In our case perforation at the time of insertion cannot be excluded, although the patient did not complain of undue pain or discomfort immediately after the procedure. This would explain the presence of the implant in the peritoneal cavity proven by pelvic X-ray 6 weeks post insertion. It does not, however, explain the complete absence of the device at laparoscopy and X-ray 3 months later. It was therefore assumed that there was some kind of predisposition to perforation. Procedures had been carried out by experienced operators, yet the uterus was penetrated twice in a high fundal position and the device was lost without trace, possibly by way of a perforation through the bowel wall and expulsion via the intestinal route.

Clinicians require a high level of suspicion when women attend with complaints soon after insertion of IUDs and IUIs, and a low threshold for initiating further investigations when threads can not be found on clinical examination is appropriate. Women should be advised of the possibility of perforation or expulsion prior to IUD/IUI insertion. They need to be taught to feel for their thread to confirm presence of the device and to attend for a check up in the event that they cannot feel their thread anymore while using an alternative method of contraception.

Statements on funding and competing interests

Funding. None.

Competing interests. None declared.

References

Trien K, Liski L, et al IUDs - An update. Population Reports. Baltimore: Johns Hopkin School of Public Health, 1995; IUDs - An update Series B, No 6: 9 Andersson K, Ryde-Blomquist E,

Chi I. What we have learned from recent IUD studies: A researcher's perspective. Contraception 1993; 48: 81-108.

Wildemeersch D, Batar I, Webb A, et al. Gynefix. The frameless intrauterine contraceptive implant - an update. Br J Family Planning 1999: 24: 149-159.

Vekemans M, Verougstraete A. Late Uterine Perforation With an Anchored IUD, the Gynefix: A Case Report. Contraception 1999; 60: 55-56.

Faculty of Family Planning and Reproductive Health Care. Continuing medical education and recertification: diplomates. London: Faculty of Family Planning and Reproductive Health Care of the RCOG, 1997 .

al-Kamil RK. Perforation of uterus by the GyneFix intrauterine contraceptive device. Int J Clin Pract 2000; 54 (2): $128-129$

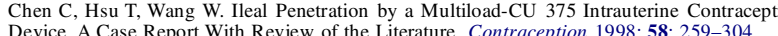

